Provided for non-commercial research and education use. Not for reproduction, distribution or commercial use.

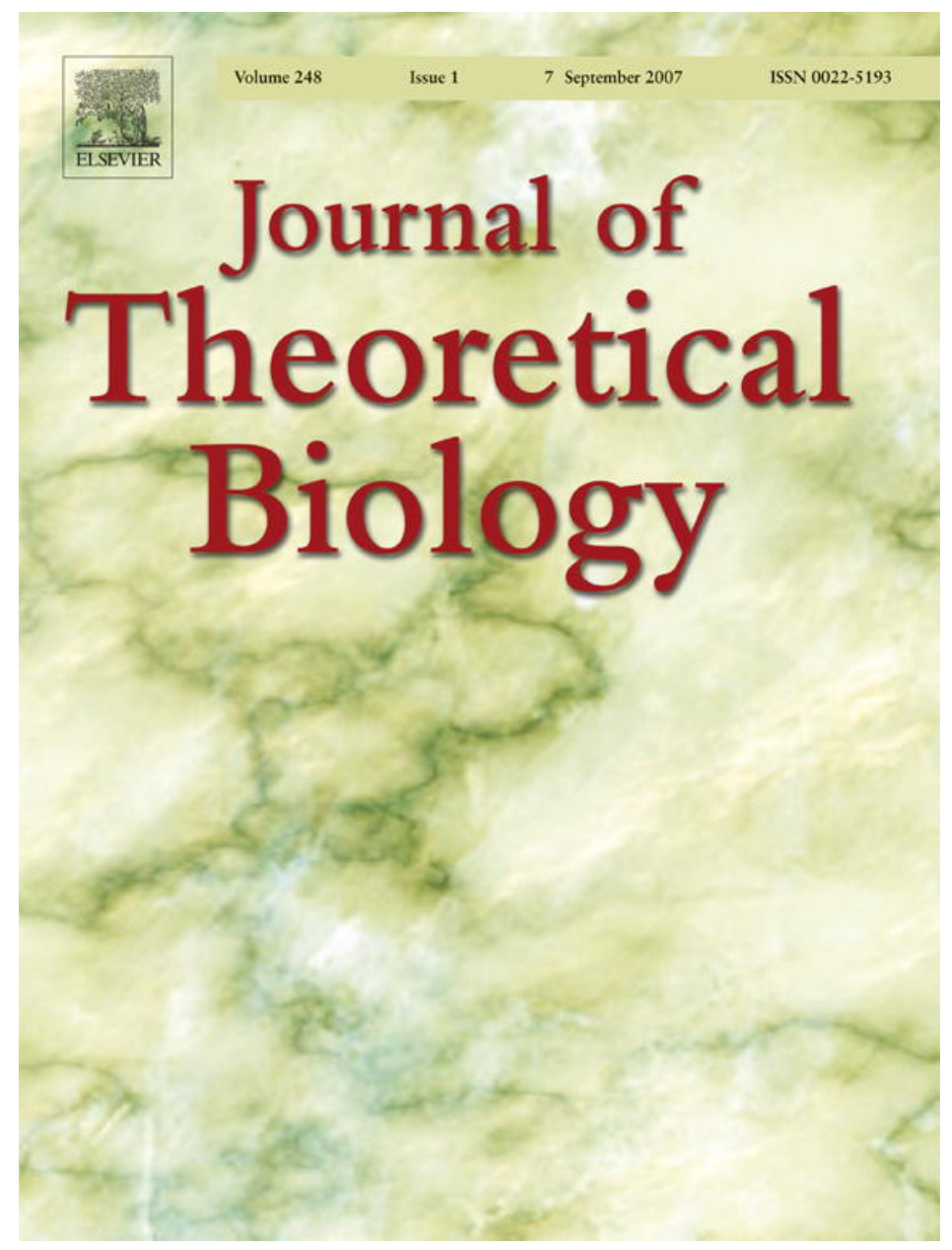

This article was published in an Elsevier journal. The attached copy

is furnished to the author for non-commercial research and education use, including for instruction at the author's institution, sharing with colleagues and providing to institution administration.

Other uses, including reproduction and distribution, or selling or licensing copies, or posting to personal, institutional or third party websites are prohibited.

In most cases authors are permitted to post their version of the article (e.g. in Word or Tex form) to their personal website or institutional repository. Authors requiring further information regarding Elsevier's archiving and manuscript policies are encouraged to visit: 


\title{
The segmentation clock in mice: Interaction between the Wnt and Notch signalling pathways
}

\author{
J.G. Rodríguez-González ${ }^{\mathrm{a}}$, M. Santillán ${ }^{\mathrm{a}, \mathrm{b}, \mathrm{g}, *}$, A.C. Fowler ${ }^{\mathrm{c}}$, Michael C. Mackey ${ }^{\mathrm{b}, \mathrm{d}, \mathrm{e}, \mathrm{f}}$ \\ ${ }^{a}$ Unidad Monterrey, CINVESTAV-IPN, Av. Cerro de las Mitras No. 2565, Col. Obispado, 64060 Monterrey NL, México \\ ${ }^{\mathrm{b}}$ Centre for Nonlinear Dynamics, McGill University, 3655 Promenade Sir William Osler, H3G 1 Y6 Montreal, Que., Canada \\ ${ }^{c}$ Mathematical Institute, University of Oxford, 24-29 St Giles, Oxford OX13LB, UK \\ ${ }^{\mathrm{d}}$ Department of Physiology, McGill University, Montreal, Que., Canada \\ ${ }^{\mathrm{e}}$ Department of Physics, McGill University, Montreal, Que., Canada \\ ${ }^{\mathrm{f}}$ Department of Mathematics, McGill University, Montreal, Que., Canada \\ ${ }^{\mathrm{g}}$ Depto de Fisica, Esc. Sup. de Física y Matemáticas, Instituto Politécnico Nacional, Edif. 9, U. P. Zacatenco, 07738 México DF, México
}

Received 4 September 2006; accepted 4 May 2007

Available online 22 May 2007

\begin{abstract}
In the last few years, the efforts to elucidate the mechanisms underlying the segmentation clock in various vertebrate species have multiplied. Early evidence suggested that oscillations are caused by one of the genes under the Notch signalling pathway (like those of the her or Hes families). Recently, Aulehla et al. [Wnt3a plays a major role in the segmentation clock controlling somitogenesis. Dev. Cell 4, 395-406] discovered that Axin2 (a gene under the Wnt3a signalling pathway) also oscillates in the presomitic mesoderm (PSM) of mice embryos and proposed some mechanisms through which the Notch and Wnt3a pathways may interact. They further suggested that a decreasing concentration of Wnt3a along the PSM may be the gradient the segmentation clock interacts with to form somites. These results were reviewed by Rida et al. [A notch feeling of somite segmentation and beyond. Dev. Biol. 265, 2-22], who introduced a complex clockwork comprising genes Hes1, Lfing (under the Notch pathway), and Axin2, as well as their multiple interactions. In the present work we develop a mathematical model based on the Rida et al. review and use it to tackle some of the questions raided by the Aulehla et al. paper: can the Axin2 feedback loop constitute a clock? Could a decreasing Wnt3a signaling constitute the wavefront, where phase is recorded and the spatial pattern laid down? What is the master oscillator?
\end{abstract}

(C) 2007 Elsevier Ltd. All rights reserved.

Keywords: Somitogenesis; Mathematical model; System biology; Computational biology; Time delays; Oscillations

\section{Introduction}

During their early developmental stages, the embryos of all vertebrates organize in three layers of cells known as the endoderm, the mesoderm, and the ectoderm (see Fig. 1A). The endoderm gives rise to the digestive, urinary and respiratory epithelia, the thymus, the thyroid, etc., while

\footnotetext{
${ }^{*}$ Corresponding author. Unidad Monterrey, CINVESTAV-IPN, Av. Cerro de las Mitras No. 2565, Col. Obispado, 64060 Monterrey NL, México. Tel.: + 528182201740 ; fax: +52818200741 .

E-mail addresses: jrodriguez@ cinvestav.mx

(J.G. Rodríguez-González), moises.santillan@mac.com (M. Santillán), fowler@maths.ox.ac.uk (A.C. Fowler),

michael.mackey@mcgill.ca (M.C. Mackey).

URL: http://homepage.mac.com//moises.santillan (M. Santillán).
}

the mesoderm gives rise to the skeleton and the muscles, as well as the circulatory and reproductive systems, etc. Finally, the ectoderm is the origin of the skin, the nervous system, and the sensory organs.

In amniote vertebrates (reptiles, birds, and mammals) and fish, the neural tube (precursor of the spinal nerve) forms from a longitudinal invagination of the endoderm. The notochord, the skeletal axis of vertebrates, forms beneath the neural tube from endodermal cells. Both the neural tube and the notochord grow from head to tail and their posterior end is attached to the so-called primitive streak, which ends at the tail bud. On either side of the neural tube lie thick bands of mesodermal cells. These bands of paraxial mesoderm are referred to as the presomitic mesoderm (PSM). As the primitive streak 
A
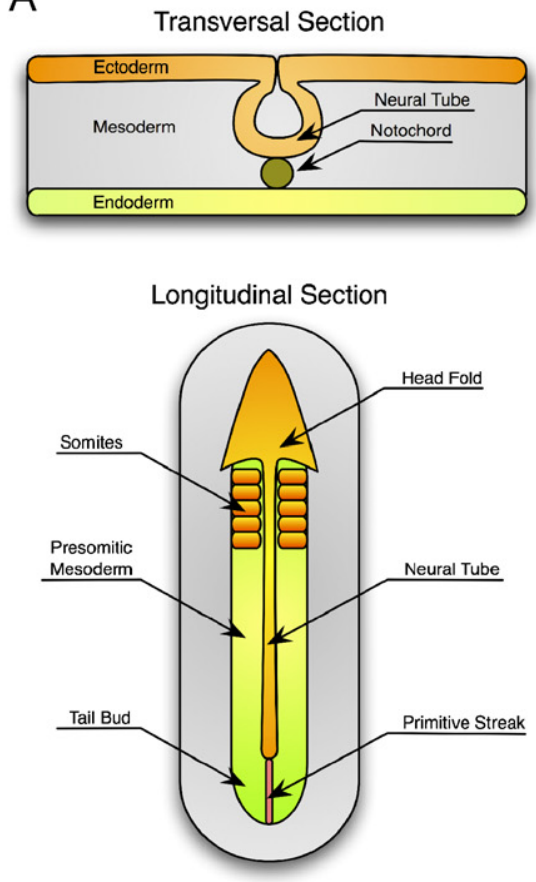

B

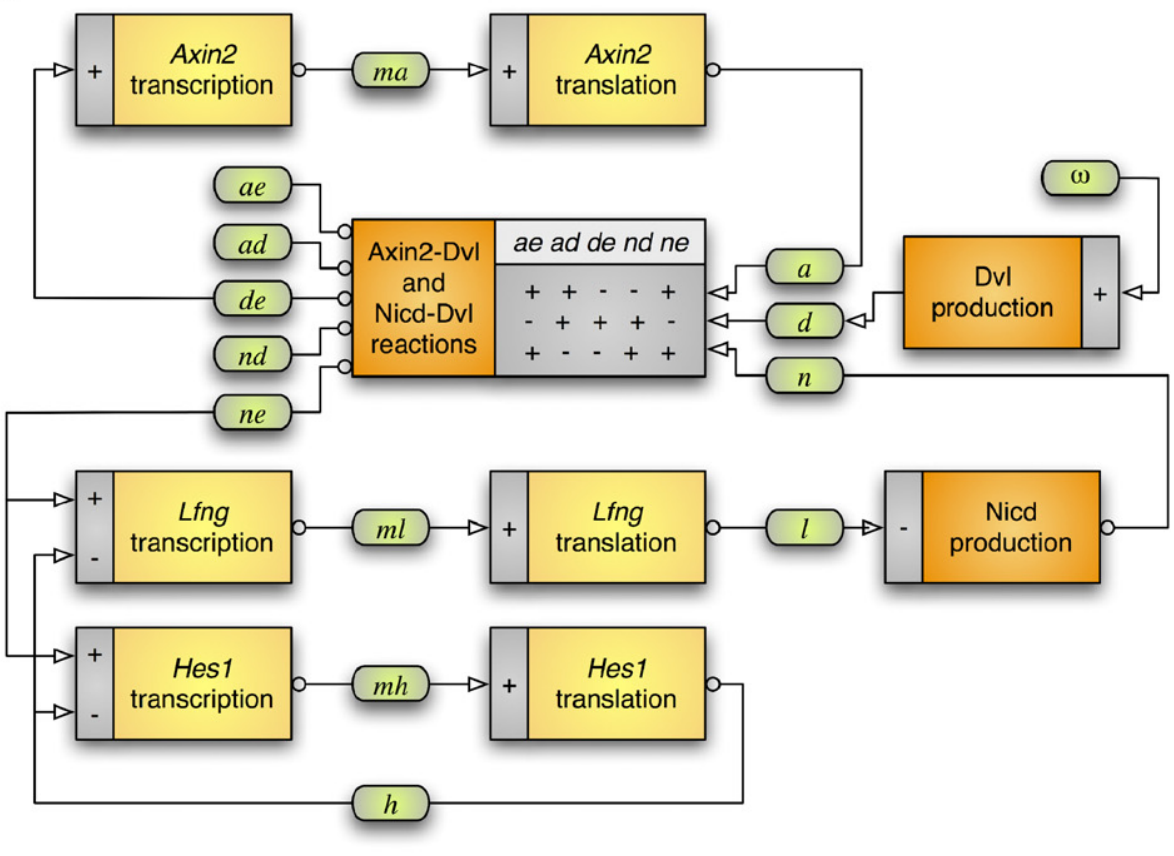

Fig. 1. (A) Diagrams showing transversal and longitudinal sections of a vertebrate embryo. The transversal section illustrates the organization of the different cell layers, as well as the neural tube and notochord development. The longitudinal section illustrates the formation and organization of somites. (B) Schematic representation of the segmentation-clock model proposed by (Rida et al., 2004) for mice. The model variables are shown enclosed in round boxes, and their meaning is as follows: $\omega$ is the normalized Wnt3a concentration; $a$, $a e$, and $a d$, respectively, denote the normalized concentration of total, free, and bound-to-Dvl Axin2; $n, n e$, and $n d$ represent the total, free, and bound-to-Dvl Nicd normalized concentrations; $d$ and $d e$ are the total and free Dvl normalized concentrations; $h$ and $l$ stand for the total normalized concentration of Hes 1 and Lfng, respectively; and $m a$, $m h$, and $m l$ represent the normalized concentrations of the Axin2, Hes1, and Lfing mRNA species. All of the processes underlying the segmentation clock are represented by rectangles. Inputs (outputs) are denoted with empty arrows (circles). Finally, plus and minus signs stand for the effect each input variable has on every output variable.

regresses, the PSM separates into blocks of cells called somites. Although somites are transient structures, they are extremely important in organizing the segmental pattern of vertebrate embryos. Somites give rise to the cells that form the vertebrate and ribs, the dermis of the dorsal skin, the skeletal muscles of the back, and the skeletal muscles of the body wall and limbs. The somites also determine the migratory paths of neural crest cells and spinal nerve axons.

Several models of somitogenesis have been put forth. However, the clock and wavefront model of Cooke and Zeeman (1976) has found widest acceptance and applicability. At the heart of the clock and wavefront modelreviewed in Pourquie (2001) - is the proposed existence of a molecular clock or biochemical oscillator within the cells of the (unsegmented) PSM. According to this model, neighbouring cells are entrained with respect to their oscillations. The clock and wavefront model also postulates the existence of a wavefront of cell change that sweeps posteriorly through the PSM, slowing and halting the oscillation, and inducing or permitting somite maturation.

The discovery in 1997 of an oscillating expression of the gene $c$-hairyl in the PSM of chick embryos provided the first molecular evidence of a segmentation clock (Palmerim et al., 1997). Meanwhile, several other cycling genes, such as Lfng, her1, c-hairy2, Hes1, and Hey2 have been identified in different species (Pourquié, 2001). Recently, a graded distribution of Fgf8 along the PSM and a role of the corresponding gene in setting the segment boundary position have been reported (Dubrulle et al., 2001). These data provide molecular evidence that a gradient plays an essential role in the segmentation process, as predicted in several models (like the clock and wavefront model). On the other hand, despite great advances in the last few years, the mechanisms by which the segmentation clock is established and by which it interacts with the gradient have not yet been completely elucidated.

During the last few years, attempts to unveil the mechanisms underlying the segmentation clock have multiplied. Early evidence suggested that oscillations are generated by one of the genes under the Notch regulatory pathway, like herl or Hesl (Rida et al., 2004). Recently, Aulehla et al. (2003) discovered Axin2 (a gene under the Wnt3a regulatory pathway) oscillations in mouse and proposed some mechanisms through which the Wnt3a and Notch pathways may interact. They further suggested that a decreasing concentration of Wnt3a along the PSM may be the gradient the segmentation clock interacts with to 
form somites. To our understanding, the results of Aulehla et al. raise the following questions:

1. Can the wiring schemes for the somitogenesis clock involving a Wnt-dependent negative feedback loop, such as that outlined in the Aulehla et al. (2003) paper, generate oscillations in principle. That is, can the Axin2 feedback loop constitute a clock.

2. Aulehla et al. (2003) proposed that a gradient of Wnt3a signaling would act much as a previously studied Fgf8 gradient is thought to, by causing the oscillations to slow and stop. Thus, a second question is: could a decreasing Wnt3a signaling constitute the wavefront, where phase is recorded and the spatial pattern laid down?

3. Finally, since previous discussion of the oscillatory mechanisms had focused on Hes genes as the source of feedback loops for the generation of instabilities, the potential existence of another feedback loop raised the question of hierarchy between the oscillatory system: what is the master oscillator? For a subsystem to be a master oscillator it should be able to cycle spontaneously, to make the other subsystems oscillate, since they are not supposed capable of generating sustained oscillations; and the whole system must oscillate with the period and the phase shifts among subsystems experimentally observed.

In the present paper, we tackle these questions from a mathematical modeling point of view. For this, we draw on previous mathematical models, which address different aspects of the segmentation clock dynamic behaviour (Lewis, 2003; Monk, 2003; Hirata et al., 2004; Bernard et al., 2006).

\section{Mathematical model of the segmentation clock}

In a recent paper, Rida et al. (2004) review the results of several labs and propose a complex molecular clockwork for the segmentation clock in mouse (mostly) and chick. This machinery, schematically presented in Fig. 1B, includes the mechanisms suggested by Aulehla et al. (2003). Below, we present a mathematical model for the Rida et al. proposed segmentation-clock architecture, in order to tackle the previously posed questions.

The model, developed in Appendixes A and B, consists of six differential delay equations and three algebraic equations, all of which are tabulated in Table 1. The meaning of the model variables is as follows: $m a, m h$, and $m l$, respectively, stand for the normalized Axin2, Hes 1 and Lfing mRNA concentrations; $a, h$, and $l$ represent the normalized concentrations of Axin2, Hes1, and Lfng proteins; $n$ is the total normalized concentration of Notch intracellular domain (Nicd) molecules; $n e$ and de stand for the normalized concentrations of free Nicd and Dvl proteins - the full name of the corresponding gene is: dishevelled, dsh homolog 1 (Drosophila); and $\omega$ is the normalized concentration of extracellular Wnt3a. The rest of the symbols in the model equations are parameters, and the notation $[x]_{\tau}$ means that variable $x$ is delayed a time $\tau$.
Table 1

The equations for the mathematical models of the segmentation clock, as developed in Appendixes A and B

$$
\begin{aligned}
& \dot{m} a=\gamma_{m a}\left(\left[\frac{d e^{n_{d}}}{d e^{n_{d}}+k_{d a}^{n_{d}}}\right]_{\tau_{m a}}-m a\right) \\
& \dot{m} h=\gamma_{m h}\left(\left[\frac{k_{h}^{n_{h}}}{k_{h}^{n_{h}}+h^{n_{h}}} \frac{n e^{n_{n}}}{n e^{n_{n}}+k_{n}^{n_{n}}}\right]_{\tau_{m h}}-m h\right) \\
& \dot{m} l=\gamma_{m l}\left(\left[\frac{k_{h}^{n_{h}}}{k_{h}^{n_{h}}+h^{n_{h}}} \frac{n e^{n_{n}}}{n e^{n_{n}}+k_{n}^{n_{n}}}\right]_{\tau_{m l}}-m l\right)
\end{aligned}
$$

$\dot{a}=\gamma_{a}\left([m a]_{\tau_{a}}-a\right)$

$\dot{h}=\gamma_{h}\left([m h]_{\tau_{h}}-h\right)$

$\dot{l}=\gamma_{l}\left([m l]_{\tau_{l}}-l\right)$

$n=\frac{\eta}{\eta+l}$

$n e=\frac{\left(1+\beta_{a} a+\beta_{n} n\right)}{\left(1+\beta_{a} a+\beta_{n} n\right)+\beta_{d} \omega} n$

$d e=\frac{1}{1+\beta_{a} a+\beta_{n} n} \omega$

As seen in Eq. (19), Axin2 mRNA production is positively regulated by free Dvl-de-. Accordingly, from Eqs. (20) and (21), production of Hesl and Lfng mRNA is negatively (positively) regulated by free Hes $1-h-$ (free Nicd-ne-). Lfng- - has a negative effect on Nicd- $n-$ production, as shown in Eq. (25). Wnt3a enhances Dvl production, and thus the normalized Dvl concentration is assumed to be equal to $\omega$. Free Axin2 and Nicd can bind free Dvl. This is accounted for by Eqs. (26) and (27), which give the normalized concentrations of free Nicd and Dvl, as functions of the total amount of Axin2, Dvl, and Nicd.

There are two opposing views about the function of Lfng in the oscillator. One, by Dale et al. (2003) from work in chick embryos, is represented in the model above: Lfng is a feedback repressor of its own signaling through interfering with Notch activation. However, the other comes from the work of many labs in mouse embryos and mammalian cell culture and indicates that Lfng is likely to directly enhance Notch activation of the Lfng and other target genes (Zhang and Gridley, 1998; Barrantes et al., 1999; Hicks et al., 2000; Cole et al., 2002; Dunwoodie et al., 2002; Zhang et al., 2002; Serth et al., 2003). To take into account the second view, we substitute Eq. (25) by

$n=\frac{l}{\eta+l}$,

which means that Lfng enhances the production of Nicd. 
There are three different time-delayed feedback regulatory loops in the model presented above. Two of themthose corresponding to the gene Hesl, which inhibits its own expression; and to the gene Axin2, whose product binds and inhibits its activator, Dvl-involve a negative feedback. The third loop can either involve a negative or a positive feedback, depending on whether the gene Lfng inhibits or enhances Nicd production (an activator of both Lfing and Hesl).

Every one of the above described regulatory loops is potentially capable of generating sustained oscillators and may be able to entrain the other two via the following interactions. Since both Axin2 and Nicd bind Dvl, an increase of Axin2 (Nicd) decreases the amount of Dvl free to bind Nicd (Axin2), and thus increases the amount of free molecules of this second substance. Thus, the expression of Axin 2 positively affects Hes 1 and $L f n g$, which are activated by Nicd. Moreover, Lfng, which alters Nicd production, affects in opposite directions the expression levels of Axin2 (because it is activated by Dvl) and Hesl (because it is activated by Nicd). Finally, Hesl directly downregulates Lfing and indirectly affects Axin2 via the Axin2-Dvl and Nicd-Dvl reactions.

\section{Results}

Ideally, all of the parameters in a mathematical model should be taken from the biochemical literature. Then, we could ask what behaviour the model shows, and therefore, whether the logic of the model is correct. Unfortunately, for the segmentation oscillator model presented above, this approach is limited.

Since at this point we are interested in the dynamic behaviour of PSM cells located in the tail bud, where the Wnt3a concentration is thought to be high (Aulehla et al., 2003), we set variable $\omega$ to its maximum value:

$\omega=1$.

Following Lewis (2003) we estimated all the time delays as follows. First, we looked for the sequence of genes Axin2, Hesl, and Lfing in the Pubmed database. The time delays due to transcription and mRNA processing prior to translation were calculated by adding the time it takes a polymerase to transcribe the gene (considering a transcription speed of 20 nucleotides per second), plus the time necessary to remove the introns (between 0.4 and $7.5 \mathrm{~min}$ per intron), plus $4 \mathrm{~min}$ to account for the time elapsed between completion of splicing and the emergence of the mature mRNA into the cytosol. The translational time delays were calculated by considering a translation speed of 6 nucleotides per second. The results of these calculations are shown in the following equation:

$\tau_{m a} \in[33.3,104.3] \mathrm{min}, \quad \tau_{a} \simeq 11.8 \mathrm{~min}$,

$\tau_{m h} \in[7.2,28.5] \mathrm{min}, \quad \tau_{h} \simeq 4.1 \mathrm{~min}$,

$\tau_{m l} \in[12.7,62.4] \mathrm{min}, \quad \tau l \simeq 3.3 \mathrm{~min}$.
Monk (2003), Giudicelli and Lewis (2004), Hirata et al. (2004), and Bernard et al. (2006) report half-life times of about $25 \mathrm{~min}$ for different proteins of the Hes family and their corresponding mRNA species. This half-life time corresponds to a degradation rate around $0.03 \mathrm{~min}^{-1}$. Since we could not find data for the Axin2 and Lfng half lives, we assumed similar degradation rates:

$$
\begin{array}{ll}
\gamma_{m a}=0.03 \mathrm{~min}^{-1}, & \gamma_{a}=0.03 \mathrm{~min}^{-1}, \\
\gamma_{m h}=0.03 \mathrm{~min}^{-1}, & \gamma_{h}=0.03 \mathrm{~min}^{-1}, \\
\gamma_{m l}=0.03 \mathrm{~min}^{-1}, & \gamma_{l}=0.03 \mathrm{~min}^{-1} .
\end{array}
$$

We were unable to find reliable experimental sources to estimate the rest of the parameters, so we decided to explore the model parameter space to ask what volumes in parameter space host what kinds of behaviours. To carry out this exploration orderly, we uncoupled the Axin2, Hes1, and Lfng subsystems and analysed them in a separate fashion. The equations for the resulting Axin2 subsystem are

$\dot{m} a=\gamma_{m a}\left(\left[\frac{d e^{n_{d}}}{d e^{n_{d}}+k_{d a}^{n_{d}}}\right]_{\tau_{m a}}-m a\right)$,

$\dot{a}=\gamma_{a}\left([m a]_{\tau_{a}}-a\right)$,

$d e=\frac{1}{1+\beta_{a} a} \omega$.

The Hes1 uncoupled subsystem equations are

$\dot{m} h=\gamma_{m h}\left(\left[\frac{k_{h}^{n_{h}}}{k_{h}^{n_{h}}+h^{n_{h}}}\right]_{\tau_{m h}}-m h\right)$,

$\dot{h}=\gamma_{h}\left([m h]_{\tau_{h}}-h\right)$.

Finally, for the Lfng uncoupled subsystem with negative feedback we have

$$
\dot{m} l=\gamma_{m l}\left(\left[\frac{n e^{n_{n}}}{n e^{n_{n}}+k_{n}^{n_{n}}}\right]_{\tau_{m l}}-m l\right),
$$

$i=\gamma_{l}\left([m l]_{\tau_{l}}-l\right)$,

$n e=\frac{1+\beta_{n} n}{1+\beta_{n} n+\beta_{d} \omega} n$,

$n=\frac{\eta}{\eta+l}$.

In the case of positive feedback, the last equation is substituted by

$n=\frac{l}{\eta+l}$.

The analysis of each uncoupled subsystem, of different pairs of coupled systems (introduced below) and of the full system, was performed by numerically solving the 
corresponding sets of differential equations with the aid of the programs MatLab and Simulink.

Bernard et al. (2006) analysed the stability of a mathematical model for the regulatory pathway of Hes1. Such model structure is similar to the one introduced above, and the time delays and degradation rates considered are similar as well. According to Bernard et al., given the time-delay and degradation-rate values, a Hill exponent larger than 4.5 is necessary to have sustained oscillations. We found that Hill exponents of the order of 7 were necessary for each of the three subsystems to oscillate spontaneously. Therefore, we set:

$n_{d}=7, \quad n_{h}=7, \quad n_{n}=7$.

We further explored the parameter space for each of the above presented subsystems. Below we present the parameter ranges for which sustained oscillations are observed in each case. To explore the parameter space of the Axin2 subsystem we fixed $\tau_{a}=11.8 \mathrm{~min}$ and took three different $\tau_{m a}$ values, within the range reported in Eq. (1). Since there are two free parameters $\left(k_{d a}\right.$ and $\left.\beta_{a}\right)$, we fixed one of them and looked for the values of the other parameter for which the subsystem shows sustained oscillations. The results are tabulated in Table 2.

For the Hes1 subsystem we set $\tau_{h}=4.1 \mathrm{~min}$ and considered three different values of $\tau_{m h}$ within the range reported in Eq. (1). In Table 3 we report the intervals of parameter $k_{h}$ for which sustained oscillations are observed, as well as the corresponding oscillation periods.

In the Lfng subsystem with negative feedback regulation, we fixed $\tau_{l}=3.3 \mathrm{~min}$ and took three different values for $\tau_{m l}$ in the interval given in Eq. (1). Then, we looked for the parameter ranges for which this subsystem presents sustained oscillations. Since there are three free parameters, we carried out this parameter-space exploration by moving only one of them at a time, while the value of the other two remained fixed. The results are reported in Table 4.

When positive feedback regulation was assumed for the Lfng subsystem, no sustained oscillations were observed whatsoever for any parameter combination.

We also analysed how different subsystems interact. Our aim was to figure out whether one of them can entrain the

Table 2

Intervals of parameters $k_{d a}$ and $\beta_{a}$ for which sustained oscillations are observed in the Axin2 subsystem, for different values of $\tau_{m a}$

\begin{tabular}{llll}
\hline$\tau_{m a}(\min )$ & $k_{d a}$ & $\beta_{a}$ & $T_{\text {osc }}(\min )$ \\
\hline 11.8 & 0.05 & {$[30,750]$} & $200 \rightarrow 270$ \\
11.8 & {$[0.03,0.6]$} & 60 & $190 \rightarrow 250$ \\
60 & 0.03 & {$[50,10,000]$} & $260 \rightarrow 360$ \\
60 & {$[0.02,0.3]$} & 100 & $260 \rightarrow 320$ \\
100 & 0.03 & {$\left[50,>1 \times 10^{5}\right]$} & $360 \rightarrow 540$ \\
100 & {$[0.01,1]$} & 150 & $350 \rightarrow 465$ \\
\hline
\end{tabular}

These intervals were calculated by varying one parameter at a time. In the last column, the change in the oscillation period $\left(T_{\text {osc }}\right)$ as the corresponding parameter increases is shown.
Table 3

$k_{h}$ intervals for which sustained oscillations are observed in the Hes1 subsystem, for different values of $\tau_{m h}$

\begin{tabular}{lll}
\hline$\tau_{m h}(\min )$ & $k_{h}$ & $T_{\text {osc }}(\mathrm{min})$ \\
\hline 7.3 & {$[0.02,0.14]$} & $115 \rightarrow 104$ \\
15 & {$[0.02,0.4]$} & $165 \rightarrow 120$ \\
25 & {$[0.01,0.6]$} & $245 \rightarrow 155$ \\
\hline
\end{tabular}

In the last column, the change in the oscillation period as $k_{h}$ increases is shown.

Table 4

Intervals of parameters $k_{n}, \beta_{n}=\beta_{d}$, and $\eta$ for which sustained oscillations are observed in the Lfng subsystem, for different values of $\tau_{m h}$

\begin{tabular}{lllll}
\hline$\tau_{m l}(\min )$ & $k_{n}$ & $\beta_{n}=\beta_{d}$ & $\eta$ & $T_{\text {osc }}(\min )$ \\
\hline 13 & 0.05 & 50 & {$[0.005,0.14]$} & $170 \rightarrow 110$ \\
13 & 0.05 & {$\left[20,>1 \times 10^{5}\right]$} & 0.1 & $115 \rightarrow 115$ \\
13 & {$[0.02,0.18]$} & 50 & 0.1 & $110 \rightarrow 125$ \\
16.7 & 0.05 & 50 & {$[0.005,0.14]$} & $200 \rightarrow 120$ \\
16.7 & 0.05 & {$\left[10,>1 \times 10^{5}\right]$} & 0.1 & $120 \rightarrow 120$ \\
16.7 & {$[0.02,0.2]$} & 50 & 0.1 & $126 \rightarrow 140$ \\
40 & 0.05 & 50 & {$[0.001,0.2]$} & $250 \rightarrow 182$ \\
40 & 0.05 & {$\left[5,>1 \times 10^{5}\right]$} & 0.1 & $180 \rightarrow 195$ \\
40 & {$[0.02,0.3]$} & 50 & 0.1 & $190 \rightarrow 225$
\end{tabular}

These intervals were calculated by varying one parameter at a time, while fixing the other two. In the last column, the change in the oscillation period as the corresponding parameter increases is shown.

others when it oscillates spontaneously. Given that the Lfng subsystem does not show sustained oscillation when it is subject to positive feedback regulation, we focused our attention to the interaction between the Axin2 and the Hes 1 subsystems. The equations for the coupled Axin2 and Hes1 subsystems are

$\dot{m} a=\gamma_{m a}\left(\left[\frac{d e^{n_{d}}}{d e^{n_{d}}+k_{d a}^{n_{d}}}\right]_{\tau_{m a}}-m a\right)$,

$\dot{m} h=\gamma_{m h}\left(\left[\frac{k_{h}^{n_{h}}}{k_{h}^{n_{h}}+h^{n_{h}}} \frac{n_{e}^{n_{n}}}{n_{e}^{n_{n}}+k_{n}^{n_{n}}}\right]_{\tau_{m h}}-m h\right)$,

$\dot{a}=\gamma_{a}\left([m a]_{\tau_{a}}-a\right)$,

$\dot{h}=\gamma_{h}\left([m h]_{\tau_{h}}-h\right)$,

$d e=\frac{1}{1+\beta_{a} a} \omega$

$n e=\frac{1+\beta_{a} a}{1+\beta_{a} a+\beta_{d} \omega}$.

To analyse this coupled system we set the degradation rates to the values given in Eq. (2), the translational delays to the values reported in Eq. (1), the Hill exponents to the values given in Eq. (3), and the rest of the parameters as 
follows:

$\tau_{m h}=15.8 \mathrm{~min}, \quad \tau_{m a}=33.3 \mathrm{~min}$,

$\omega=1, \quad k_{h}=0.18$,

$k_{n}=0.14, \quad k_{d a}=0.3$,

$\beta_{a}=60, \quad \beta_{d}=100$

With these parameter values, the Axin2 and Hes1 subsystems oscillate spontaneously and have oscillation periods of about $240 \mathrm{~min}$ and $120 \mathrm{~min}$, respectively.

To test whether Axin2 is capable of recruiting the Hes1 subsystem, we modified - one at a time - the parameters corresponding to Hes1 to stop it from oscillating; then, the behaviour of the coupled system was analysed numerically. Hes1 oscillations were stopped by either setting $k_{h}=0.6$, or by decreasing $n_{h}=n_{n}=4$. In both cases, Axin 2 made Hes 1 resonate, and both subsystems oscillated with a period of $240 \mathrm{~min}$. The oscillations of Axin 2 and Hes1 were out of phase by $90^{\circ}$, approximately.

Given that Hes1 does not affect the expression of Axin2 in the Axin2-Hes1 coupled system, Axin2 cannot be entrained by Hes1 when Axin2 spontaneous oscillations do not exist.

In conclusion, with a proper choice of parameters, Axin2 can entrain Hes1, but the coupled-system oscillation period is always close to that of Axin2, which is twice as large as the period experimentally observed in mice. Moreover, the phase shift between the Axin2 and the Hes1 oscillations is half as long as the experimental value (Aulehla et al., 2003).

We found that the oscillation period of all three uncoupled subsystems is quite sensitive to changes in the time delays, as well as to changes in the mRNA and protein degradation rates. In particular, the Axin2 oscillation period decreases as the corresponding degradation rates increase: when $\gamma_{m a}=\gamma_{a}=0.18 \mathrm{~min}^{-1}$, the Axin2 oscillation period is about $120 \mathrm{~min}$. With these increased degradation rates, the Axin2 subsystem shows sustained oscillations for $n_{d} \geqslant 2$. We repeated the numerical experiments described in the previous paragraph considering the new degradation rates and found again that Axin2 can entrain Hes1 for various choices of parameter values. In this case, the oscillation period is of the order of $2 \mathrm{~h}$, and the phase shift between the Axin 2 and Hes 1 oscillations is about $180^{\circ}$, in agreement with the experimental observations of Aulehla et al. (2003).

After studying the behaviour of the Axin2-Hes1 coupled system, we returned to the full model to analyse the influence of Lfng. For this, we used the parameter values tabulated in Table 5. With these parameter values, both Axin2 and Hes1 subsystems oscillate spontaneously with a period of about 120 min, while the Lfng subsystem does not show sustained oscillations, even if it is subject to negative feedback.

The model dynamic behaviour was analysed by setting $n_{d}=1\left(n_{h}=4\right)$ to avoid spontaneous oscillations in the Axin2 (Hes1) subsystem; then, the model equations were numerically solved to see whether the oscillations of Hes 1
Table 5

Parameter values for the full model

\begin{tabular}{ll}
\hline$\tau_{m a}=33.3 \mathrm{~min}$ & $\tau_{a}=11.8 \mathrm{~min}$ \\
$\tau_{m h}=15.8 \mathrm{~min}$ & $\tau_{h}=4.1 \mathrm{~min}$ \\
$\tau_{m l}=16.7 \mathrm{~min}^{-1}$ & $\tau l=3.3 \mathrm{~min}$ \\
$\gamma_{m a}=0.18 \mathrm{~min}^{-1}$ & $\gamma_{a}=0.18 \mathrm{~min}^{-1}$ \\
$\gamma_{m h}=0.03 \mathrm{~min}^{-1}$ & $\gamma_{h}=0.03 \mathrm{~min}^{-1}$ \\
$\gamma_{m l}=0.03 \mathrm{~min}^{-1}$ & $\gamma_{l}=0.03 \mathrm{~min}^{-1}$ \\
$k_{d a}=0.3$ & $k_{h}=0.18$ \\
$k_{n}=0.14$ & $\eta=0.1$ \\
$n_{d}=4$ & $n_{h}=7$ \\
$n_{n}=2$ & $\beta_{d}=5$ \\
$\beta_{a}=10$ & $\beta_{n}=10$ \\
$\omega=1$ & \\
\hline
\end{tabular}

(Axin2) can entrain the other two subsystems. We repeated these experiments considering both positive and negative feedback regulation in the Lfng pathway. According to our results, when negative feedback is taken into consideration, both Axin2 and Hes1 can play the role of master oscillator; each can make the whole system oscillate, with Hes1 and Lfng oscillations in phase between them and out of phase by $180^{\circ}$ from the oscillations of Axin 2 (see Figs. $2 \mathrm{~A}$ and B). Contrarily, when positive feedback is included in the Lfng subsystem, Hes1 and Lfng never oscillate; Axin2 can oscillate, but only if it is setup as the master oscillator.

We corroborated that positive feedback in the Lfng subsystem eliminates oscillations of Hes1 by coupling this two systems together and numerically solving the resulting differential equations. For all the parameter combinations we tested, neither Hes1 nor Lfng oscillate when they are coupled, even if the Hes1 subsystem shows sustained oscillations by itself. On the other hand, when negative Lfng feedback is considered, we were able to find various parameter sets for which Hes1 entrains Lfng, and both subsystems oscillate in phase.

Wnt3a enhances Dvl production, which in turn activates Axin2. Thus, a decrease on the Wnt3a level has the effect of down-regulating the gene Axin2 and may even stop the corresponding subsystem from oscillating. Aulehla et al. (2003) demonstrated this behaviour experimentally and further suggested that a decreasing Wnt3a concentration along the PSM may be the gradient the segmentation clock interacts with to form somites. By carrying out numeric simulations we tested that Axin2 oscillation arresting can be fully accounted for by reduction of Dvl levelsconcomitant with Wnt3a-concentration decrease. However, the Hes1 and Lfng subsystems continue cycling if Axin2 is not the master oscillator. In any case, oscillations damp out slowly as the Wnt3a concentration decreases, instead of suddenly locking their phase as demanded by the clock and wavefront model (see Figs. 2C and D).

\section{Concluding remarks}

During the last years, the attempts to uncover the molecular mechanisms of the somitogenesis segmentation-clock 

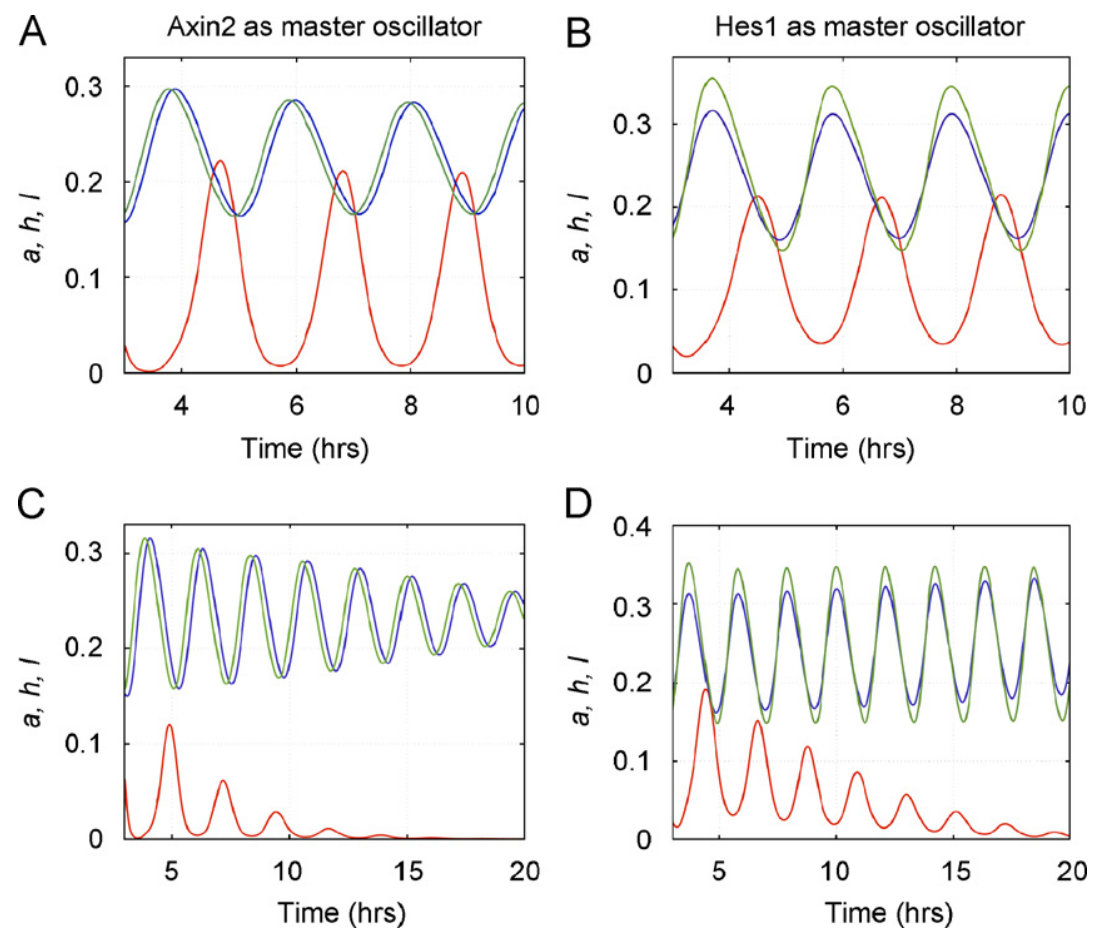

Fig. 2. Simulations, carried out under different conditions, of the segmentation-clock dynamics, using the model introduced in this paper. To calculate the plots in the left column, the Axin2 subsystem was set as the master oscillator, while for the right column plots the master oscillator is Hes1. In (A) and (B) we exemplify how, according to the model, the Axin2 and Hes1 subsystems can both be the master oscillator. In each case, the master oscillator is able to entrain the other two subsystems. Furthermore, Hes1 (green) and Lfng (blue) oscillate in phase between them, and out of phase by about half-a-cycle from the Axin2 (red) subsystem. In (C) and (D), the effect of a linearly decreasing Wnt3a concentration is shown when either Axin2 or Hes1 is the master oscillator.

have multiplied. Early experimental evidence suggested that oscillations in this system are generated by one of the genes under the Notch regulatory pathway, like herl or Hes1. Aulehla et al. discovered recently that the Axin2 (a gene under the Wnt3a regulatory pathway) expression level also oscillates in mice embryos. They further proposed some interaction mechanisms between the Wnt3a and Notch pathways, which may explain synchronization of the Axin2, Hes1, and Lfng oscillations. In this paper we developed a mathematical model for the segmentation clock clockwork proposed by Rida et al. (2004)—which incorporates the mechanisms discovered by Aulehla et al.- to explore some of the questions raised by the paper of Aulehla et al.

In our model, three time-delayed feedback regulatory loops can be identified; they are associated with the Axin2, Hes1, and Lfing genes. The Axin2 and Hes1 loops involve negative feedbacks, while regulation in the Lfng loop can be achieved through either negative or positive feedback. By exploring each subsystem's parameter space we found that the Axin2 and Hes1 subsystems can oscillate spontaneously. Furthermore, the Lfng subsystem can only generate sustained oscillations when it is subject to negative feedback regulation.

We were able to estimate all time delays, as well as the degradation rates associated with the Hes1 mRNA and protein species. Given that the corresponding half-time lives are of the same order of magnitude as the time delays, we found - in agreement with previous studies - that quite large Hill exponents are required in the corresponding regulatory function to generate sustained oscillations.

According to our results, Axin2 can entrain the Hes1 subsystem for some parameter ranges when they are coupled together. Nevertheless, the oscillation period is always close to the Axin2 natural period, which is twice as large as that experimentally observed in mice. Moreover, the phase shift between Axin2 and Hes1 oscillations is about one quarter of a cycle, in contrast with the half-acycle experimentally observed phase shift.

Given that the gene Axin2 is quite long (30418 base pairs) and has a large number of introns (10), the associated time delays are three to four times longer than those of Hes1 and Lfng. In consequence, if the Axin2 mRNA and protein species have half-time lives comparable to those of Hes1, its oscillation period is about twice as long as that experimentally observed in the mouse segmentation clock. In order for Axin2 to oscillate with the right period (around $120 \mathrm{~min}$ ), either by itself or coupled with Hes1, its mRNA and protein half lives must be about $4 \mathrm{~min}$.

If the Axin2 transcription and translation rates are not high enough, the mRNA and protein short half lives would give rise to very low counts of these molecules. Then, both the mRNA and protein levels would present large fluctuations around the values predicted by the differential-equation model. The above considerations make necessary to check the sensitivity of the model to stochastic 
effects. However, the molecule counts of all chemical species are required to perform this analysis properly (given that the amplitude of the random fluctuations increases as the number of molecules decrease), and all the corresponding variables in the present model are normalized. On the other hand, many more parameters (which cannot be estimated from experimental results) would have to be included in order to have non-normalized variables, increasing the (already large) parameter-space dimension. From these reasons we conclude that, although necessary, the above mentioned stochastic fluctuation analysis is not feasible at this time. In any case, the possibility that Axin2 oscillations are very irregular due to random fluctuations makes it a less likely candidate to be the master oscillator.

Other than the issued discussed in the previous paragraph, our simulations do not provide any information regarding whether Axin2 or Hes1 is the master oscillator in the segmentation clock of mouse. Each of these systems, when oscillating spontaneously, can make the whole system oscillate with the right period, as well as with the right phase shift between the Axin2 and the Hes1 and Lfng subsystems. On the other hand, according to our results, positive feedback regulation in the Lfng subsystem is not possible because it eliminates Hes1 and Lfng oscillations, even if the Hes1 subsystem can oscillate spontaneously by itself.

The Axin2-Wnt3a interaction mechanisms proposed by Aulehla et al. (2003) cannot explain somite formation because oscillations damp out as $\omega$ decreases below a given threshold, instead of their phase being suddenly locked. This implies that all the cells in the PSM would eventually have the same Axin2, Hes1, and Lfng levels, and therefore, that no stripes of gene activity, and so no somites, would be formed. The whole point of the wavefront is that it records the different phase of oscillators across a spatial dimension as they exit cyclic behaviour.

\section{Acknowledgements}

This research was supported by the Natural Sciences and Engineering Research Council (NSERC Grant OGP0036920, Canada), MITACS (CANADA), CONACyT (MEXICO), COFAA-IPN (MEXICO), EDI-IPN (MEXICO), the Mexican Academy of Sciences, and the Royal Society of London, and partially carried out while M.S. was visiting University of Oxford in January 2006. We thank the anonymous referees whose comments greatly helped us to improve this paper.

\section{Appendix A. Model development}

Assume that the different processes involved in the segmentation-clock regulatory pathway can be classified, according to their characteristic time, in very fast, fast, and slow processes. The fastest ones being the reactions leading to the formation of Axin2-Dvl and Nicd-Dvl complexes. Then, the fast processes are those involved in the production and degradation of Dvl and Nicd molecules.
Finally, transcription and translation of genes Axin2, Hesl, and $L f n g$ are the slowest processes.

\section{A.1. Very fast processes}

Consider the very fast processes. Let $\mathrm{Ae}, \mathrm{De}$, and $\mathrm{Ne}$, respectively, denote the cytosolic concentration of free (not bound) Axin2, Dvl, and Nicd. These three substances can combine according to the following reactions:

$A e+D e \stackrel{K_{A D}}{\rightleftharpoons} A d$ and $N e+D e \stackrel{K_{N D}}{\rightleftharpoons} N d$.

The equilibrium equations for these reactions are

$A e D e=K_{A D} A d \quad$ and $\quad N e D e=K_{N D} N d$.

Consider a constant total concentration of Axin2, Dvl, and Nicd, and respectively, denote them as $A, D$, and $N$. From their definition, these quantities obey the following relations:

$A e+A d=A, \quad N e+N d=N \quad$ and

$D e+A d+N d=D$.

Eqs. (4) and (5) constitute a complete set of algebraic equations for variables $A e, D e, N e, A d$, and $N d$. It follows after a little algebra that variable $D e$ satisfies the following equation:

$A \frac{D e}{K_{A D}+D e}+N \frac{D e}{K_{N D}+D e}=D-D e$.

Assume that $K_{A D}=K_{N D}=K_{D}$ and that the value of $D e$ that satisfies Eq. (6) is such that the Michaelis-Menten-like factors in the equation left-hand side are in their linear regime. From these assumptions, Eq. (6) can be rewritten as

$\frac{A+N}{K_{D}} D e=D-D e$.

This last equation can be straightforwardly solved rendering

$D e=\frac{K_{D}}{K_{D}+A+N} D$.

It is easy to show from this last result and Eqs. (4) and (5) that the approximated solutions for $\mathrm{Ae}$ and $\mathrm{Ne}$ are given by

$$
\begin{aligned}
& A e=\frac{\left(K_{D}+A+N\right)}{\left(K_{D}+A+N\right)+D} A \text { and } \\
& N e=\frac{\left(K_{D}+A+N\right)}{\left(K_{D}+A+N\right)+D} N .
\end{aligned}
$$

\section{A.2. Fast processes}

Consider now the processes leading to the production and degradation of Nicd. The trans-membrane protein Notch becomes non-susceptible to releasing its intracellular domain when bound by Lfng. Let $\mathscr{N} s, \mathscr{N} n$, and $\mathscr{N}$, respectively, denote the concentrations of susceptible, non-susceptible, and total Notch proteins. Accordingly, let $L$ represent the Lfng concentration. As described above,

$$
\mathscr{N} s+L \stackrel{K_{L N}}{\rightleftharpoons} \mathscr{N} n
$$


The equilibrium equation for this reaction is: $\mathscr{N} s L=$ $K_{L N} \mathscr{N} n$. Furthermore, under the assumption that production of Notch proteins is regulated in such a way that the concentration of these proteins remains constant, we have the following conservation condition: $\mathscr{N} s+\mathscr{N} n=\mathscr{N}$. By combining these last results we obtain

$\mathscr{N} s=\frac{K_{L N}}{K_{L N}+L} \mathscr{N}$.

$\mathscr{N} s$ is the substrate of a catalytic reaction that releases the Nicd $(N)$. Therefore, the production rate for $N$ obeys a Michaelis-Menten-like function:

$\dot{N}_{\text {prod }}=k_{N} \frac{\mathscr{N} s}{\mathscr{N} s+K_{N S}}=k_{N} \frac{K_{L N} \mathscr{N} / K_{N S}}{K_{L N}\left(\mathscr{N}+K_{N S}\right) / K_{N S}+L}$

with $k_{N}$ the maximum production rate of Nicd molecules. Under the assumption that $\mathscr{N} \gg K_{N S}$, the above equation can be approximated by $\dot{N}_{\text {prod }}=k_{N} K_{N} /\left(K_{N}+L\right)$, with $K_{N}=K_{L N} \mathscr{N} / K_{N S}$, and the equation governing the dynamics of $N$ comes out to be

$\dot{N}=k_{N} \frac{K_{N}}{K_{N}+L}-\gamma_{N} N$,

where $\gamma_{N}$ is the Nicd degradation rate.

Since the production of intracellular Dvl $(D)$ is catalysed by extracellular Wnt3a $(W)$, the dynamic evolution of $D$ is given by

$\dot{D}=k_{D} W-\gamma_{D} D$.

In the equation above, $k_{D}$ represents the rate of $D$ molecules production per unit of extracellular Wnt3a concentration, while $\gamma_{D}$ is the degradation rate for $D$.

If the dynamics of $D$ and $N$ are fast enough, quasi-steady assumptions for Eqs. (10) and (11) lead to

$N=\alpha_{N} \frac{K_{N}}{K_{N}+L} \quad$ and $\quad D=\alpha_{D} W$

where $\alpha_{N}=k_{N} / \gamma_{N}$ and $\alpha_{D}=k_{D} / \gamma_{D}$.

\section{A.3. $m R N A$ dynamics}

Free Nicd enter the nucleus and bind a transcription factor, transforming it from a repressor into an activator for both Hesl and Lfing. That is, the expression level of genes Hesl and Lfng is a growing function of $\mathrm{Ne}$. Moreover, Hesl can bind DNA to repress transcription of genes Hesl and Lfng by binding a specific DNA site. Following Lewis (2003), Monk (2003), Hirata et al. (2004), Bernard et al. (2006) we assume that these interactions can be modelled via Hill-type equations. Thus, the dynamics of the mRNA species corresponding to Hesl $(M h)$ and Lfing $(M l)$ are governed by

$$
\begin{aligned}
& \dot{M} h=k_{M H}\left[\frac{K_{H H}^{n_{h}}}{K_{H H}^{n_{h}}+H^{n_{h}}} \frac{N e^{n_{n}}}{N e^{n_{n}}+K_{N H}^{n_{n}}}\right]_{\tau_{M H}}-\gamma_{M H} M h, \\
& \dot{M} l=k_{M L}\left[\frac{K_{H L}^{n_{h}}}{K_{H L}^{n_{h}}+H^{n_{h}}} \frac{N e^{n_{n}}}{N e^{n_{n}}+K_{N L}^{n_{n}}}\right]_{\tau_{M L}}-\gamma_{M L} M l .
\end{aligned}
$$

In Eqs. (13) and (14) $k_{M H}$ and $k_{M L}$ are the maximum transcription initiation rates for the Hesl and Lfng genes, respectively; $\gamma_{M H}$ and $\gamma_{M L}$ denote the corresponding degradation rates; $\tau_{M H}$ and $\tau_{M L}$ correspond to the times necessary to produce completely functional Hesl and Lfing mRNA molecules, respectively; $n_{h}$ and $n_{n}$ are Hill exponents to be determined; and $[\cdots]_{\tau}$ means that all variables inside the square brackets are delayed a time $\tau$.

Gene Axin2 is activated by Dvl. It is not clear if Dvl is an activator itself or it has to bind a transcription factor to turn it into an activator. In either case, we can assume that the corresponding regulatory function is also a Hill-type function. Thus, the dynamic equation for Axin2 mRNA is

$\dot{M} a=k_{M A}\left[\frac{D e^{n_{d}}}{D e^{n_{d}}+K_{D A}^{n_{d}}}\right]_{\tau_{M A}}-\gamma_{M A} M a$,

where $k_{M A}$ is the maximum transcription initiation rate for the Axin 2 gene, $\gamma_{M A}$ denotes the corresponding degradation rate, $n_{d}$ is a Hill exponent, and $\tau_{M A}$ corresponds to the time necessary to produce a completely functional Axin2 mRNA molecule. In Eq. (15) we have taken into consideration that degradation Axin 2 mRNA is driven by the Wnt3a signalling pathway, as well as that of Axin2 polypeptides.

The supposition that the concentrations of Nicd, Hes1, and Dvl in the nucleus are proportional at any time to the corresponding concentrations in the cytosol is underlying Eqs. (13)-(15).

\section{A.4. Protein dynamics}

Assume that all mRNA species are immediately transported out of the nucleus as soon as they are completely processed. Then, the dynamic equations for the concentrations of proteins Axin2 $(A)$, Hes1 $(H)$, and Lfng $(L)$ in the cytosol are

$\dot{A}=k_{A}[M a]_{\tau_{A}}-\gamma_{A} A$,
$\dot{H}=k_{H}[M h]_{\tau_{H}}-\gamma_{H} H$,
$\dot{L}=k_{L}[M l]_{\tau_{L}}-\gamma_{L} L$.

In Eqs. (16)-(18), $k_{A}, k_{H}$, and $k_{L}$, respectively represent the maximum translation initiation rates for Axin2, Hes1, and $L f n g$ transcripts; $\gamma_{A}$ is the $A$ degradation rate, while $\gamma_{H}$ and $\gamma_{L}$ are the degradation rates for $H$ and $L$; and $\tau_{A}, \tau_{H}$, and $\tau_{L}$ the times necessary to fully assembly the respective proteins. The fact that $A$ degradation is driven by the Wnt3a pathway was taken into consideration in Eq. (16).

\section{Appendix B. Normalization}

In summary, the equations of the model developed in the previous section are

$\dot{M} a=k_{M A}\left[\frac{D e^{n_{d}}}{D e^{n_{d}}+K_{D A}^{n_{d}}}\right]_{\tau_{M A}}-\gamma_{M A} M a$, 


$$
\begin{aligned}
& \dot{M} h=k_{M H}\left[\frac{K_{H H}^{n_{h}}}{K_{H H}^{n_{h}}+H^{n_{h}}} \frac{N e^{n_{n}}}{N e^{n_{n}}+K_{N H}^{n_{n}}}\right]_{\tau_{M H}}-\gamma_{M H} M h, \\
& \dot{M} l=k_{M L}\left[\frac{K_{H L}^{n_{h}}}{K_{H L}^{n_{h}}+H^{n_{h}}} \frac{N e^{n_{n}}}{N e^{n_{n}}+K_{N L}^{n_{n}}}\right]_{\tau_{M L}}-\gamma_{M L} M l, \\
& \dot{A}=k_{A}[M a]_{\tau_{A}}-\gamma_{A} A, \\
& \dot{H}=k_{H}[M h]_{\tau_{H}}-\gamma_{H} H, \\
& \dot{L}=k_{L}[M l]_{\tau_{L}}-\gamma_{L} L .
\end{aligned}
$$

The following definitions are required to make the above system of delay differential equations complete:

$$
\begin{aligned}
& N=\alpha_{N} \frac{K_{N}}{K_{N}+L}, \\
& D=\alpha_{D} W, \\
& N e=\frac{\left(K_{D}+A+N\right)}{\left(K_{D}+A+N\right)+D} N, \\
& D e=\frac{K_{D}}{K_{D}+A+N} D .
\end{aligned}
$$

Given that there is no enough experimental information to estimate all of the parameters in the above equations it is convenient to normalize them. This will not reduce the number of parameters but will allow us to work with relative instead of absolute values. The normalized model equations read as follows:

$$
\begin{aligned}
& \dot{m} a=\gamma_{m a}\left\{\left[\frac{d e^{n_{d}}}{d e^{n_{d}}+k_{d a}^{n_{d}}}\right]_{\tau_{m a}}-m a\right\}, \\
& \dot{m} h=\gamma_{m h}\left\{\left[\frac{k_{h}^{n_{h}}}{k_{h}^{n_{h}}+h^{n_{h}}} \frac{n e^{n_{n}}}{n e^{n_{n}}+k_{n}^{n_{n}}}\right]_{\tau_{m h}}-m h\right\}, \\
& \dot{m} l=\gamma_{m l}\left\{\left[\frac{k_{h}^{n_{h}}}{k_{h}^{n_{h}}+h^{n_{h}}} \frac{n e^{n_{n}}}{n e^{n_{n}}+k_{n}^{n_{n}}}\right]_{\tau_{m l}}-m l\right\}, \\
& \dot{a}=\gamma_{a}\left\{[m a]_{\tau_{a}}-a\right\}, \\
& \dot{h}=\gamma_{h}\left\{[m h]_{\tau_{h}}-h\right\}, \\
& \dot{l}=\gamma_{l}\left\{[m l]_{\tau_{l}}-l\right\}, \\
& n e=\frac{\eta}{\eta+l}, \\
& n=\frac{\left(1+\beta_{a} a+\beta_{n} n\right)}{\left.n+\beta_{n} n\right)+\beta_{d} \omega} n,
\end{aligned}
$$

$d e=\frac{1}{1+\beta_{a} a+\beta_{n} n} \omega$.

The variables and parameters in the normalized equations are defined as follows:

$$
\begin{aligned}
& m a=\frac{W_{\max } \gamma_{M A}}{k_{M A}} M a, \quad m h=\frac{\gamma_{M H}}{k_{M H}} M h, \\
& m l=\frac{\gamma_{M L}}{k_{M L}} M l, \\
& a=\frac{W_{\max } \gamma_{M A} \gamma_{A}}{k_{M A} k_{A}} A, \quad h=\frac{\gamma_{M H} \gamma_{H}}{k_{M H} k_{H}} H, \\
& l=\frac{\gamma_{M L} \gamma_{L}}{k_{M L} k_{L}} L \\
& \gamma_{m a}=\gamma_{M A} W_{\max }, \quad \gamma_{m h}=\gamma_{M H}, \quad \gamma_{m l}=\gamma_{M L}, \\
& \gamma_{a}=\gamma_{A} W_{\text {max }}, \quad \gamma_{h}=\gamma_{H}, \quad \gamma_{l}=\gamma_{L}, \\
& \tau_{m a}=\tau_{M A}, \quad \tau_{m h}=\tau_{M H}, \quad \tau_{m l}=\tau_{M L}, \\
& \tau_{a}=\tau_{A} W_{\max }, \quad \tau_{h}=\tau_{H}, \quad \tau l=\tau_{L}, \\
& k_{d a}=\frac{K_{D A}}{\alpha_{D} W_{\max }}, \quad k_{h}=\frac{k_{M H} k_{H}}{\gamma_{M H} \gamma_{H}} K_{H H}, \quad k_{n}=\frac{K_{N L}}{\alpha_{N}}, \\
& \beta_{a}=\frac{k_{M A} k_{A}}{W_{\max } \gamma_{M A} \gamma_{A} K_{D}}, \quad \beta_{n}=\frac{\alpha_{N}}{K_{D}}, \quad \beta_{d}=\frac{\alpha_{D} W_{\max }}{K_{D}}, \\
& \omega=\frac{D_{T}}{\alpha_{D} W_{\max }}, \quad \eta=\frac{k_{M L} k_{L}}{\gamma_{M L} \gamma_{L}} K_{N} .
\end{aligned}
$$

It follows from the above definitions that all variables and all parameters, except the degradation rates and the time delays, are non-dimensional. The degradation rates have units of $\mathrm{min}^{-1}$ and the time delays are expressed in min. $W_{\max }$ is the maximum value the Wnt3a concentration can attain. The assumption that the normalized transcription rate of genes Hesl and Lfng is regulated identically is underlying the normalized model.

\section{References}

Aulehla, A., Wehrle, C., Brand-Saberi, B., Kelmer, R., Gossler, A., Kanzler, B., Herrmann, B.G., 2003. Wnt3a plays a major role in the segmentation clock controlling somitogenesis. Dev. Cell 4, 395-406.

Barrantes, I.B., Elia, A.J., Wunsch, K., Hrabe de Angelis, M.H., Mak, T.W., Rossant, J., Conlon, R.A., Gossler, A., de la Pompa, J.L., 1999. Interaction between Notch signalling and Lunatic fringe during somite boundary formation in the mouse. Curr. Biol. 9, $470-480$.

Bernard, S., Cajavec, B., Pujo-Menjouet, L., Mackey, M.C., Herzel, H., 2006. Modelling transcriptional feedback loops: the role of Gro/TLE1 in Hes1 oscillations. Phil. Trans. R. Soc. London Ser. A 364, $1155-1170$.

Cole, S.E., Levorse, J.M., Tilghan, S.M., Vogt, T.F., 2002. Clock regulatory elements control cyclic expression of Lunatic fringe during somitogenesis. Dev. Cell. 3, 75-84. 
Cooke, J., Zeeman, E.C., 1976. A clock and wavefront model for control of the number of repeated structure during animal morphogenesis. J. Theor. Biol. 58, 455-476.

Dale, J.K., Maroto, M., Dequeant, M.L., Malapert, P., McGrew, M., Pourquie, O., 2003. Periodic notch inhibition by lunatic fringe underlies the chick segmentation clock. Nature 421, 275-278.

Dubrulle, J., McGrew, M.J., Pourquié, O., 2001. FGF signalling controls somite boundary position and regulates segmentation clock control of spatiotemporal Hox gene activation. Cell 106, 219-232.

Dunwoodie, S.L., Clements, M., Sparrow, D.B., Sa, X., Conlon, R.A., Beddington, R.S., 2002. Axial skeletal defects caused by mutation in the spondylocostal dysplasia/pudgy gene Dll3 are associated with disruption of the segmentation clock within the presomitic mesoderm. Development 129, 1795-1806.

Giudicelli, F., Lewis, J., 2004. The vertebrate segmentation clock. Curr. Opin. Genet. Dev. 14, 407-414.

Hicks, C., Johnston, S.H., di Sibio, G., Collazo, A., Vogt, T.F., Weinmaster, G., 2000. Fringe differentially modulates Jagged 1 and Deltal signalling through Notch1 and Notch2. Nat. Cell Biol. 2, 515-520.

Hirata, H., Bessho, Y., Kokubu, H., Masamizu, Y., Yamada, S., Lewis, J., Kageyama, R., 2004. Instability of Hes7 protein is crucial for somite segmentation clock. Nat. Genet. 36, 750-754.
Lewis, J., 2003. Autoinhibition with transcriptional delay: a simple mechanism for the zebrafish somitogenesis oscillator. Curr. Biol. 13, 1398-1408.

Monk, N.A.M., 2003. Oscillatory expression of Hes1, p53, and $\mathrm{NF}-\kappa \mathrm{B}$ driven by transcriptional time delays. Curr. Biol. 13, 1409-1413.

Palmerim, I., Henrique, D., Ish-Horowicz, D., Pourquie, O., 1997. Avian hairy gene expression identifies a molecular clock linked to vertebrate segmentation and somitogenesis. Cell 91, 639-648.

Pourquié, O., 2001. Vertebrate somitogenesis. Ann. Rev. Cell. Dev. Biol. 17, 311-350.

Rida, P.C.G., Minh, N.L., Jiang, Y.-J., 2004. A notch feeling of somite segmentation and beyond. Dev. Biol. 265, 2-22.

Serth, K., Schuster-Gossler, K., Cordes, R., Gossler, A., 2003. Transcriptional oscillation of lunatic fringe is essential for somitogenesis. Genes Dev. 17, 912-925.

Zhang, N., Gridley, T., 1998. Defects in somite formation in lunatic fringe-deficient mice. Nature 394, 374-377.

Zhang, N., Norton, C.R., Gridley, T., 2002. Segmentation defects of Notch pathway mutants and absence of a synergistic phenotype in lunatic fringe/radical fringe double mutant mice. Genesis 33, $21-28$. 